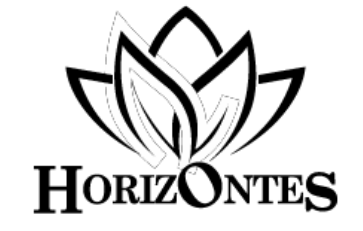

www.revistahorizontes.org
Horizontes. Revista de Investigación en Ciencias de la Educación Https://doi.org/10.33996/revistahorizontes.v2i7.54 julio - septiembre, 2018 Volumen 2 / No. 7 pp. $171-180$

\title{
Programa educativo alternativo para los estudiantes de la escuela "Juventud del Sur"
}

\section{Alternative educational program for students of the "Youth of the South" school}

\author{
Coromoto Lugo \\ chiquiclugo@gmail.com \\ Universidad de Carabobo, Venezuela \\ Miguel Chacón \\ miguelch9915@gmail.com
}

\section{RESUMEN}

El presente artículo tuvo como objetivo proponer un programa educativo alternativo a los estudiantes de la escuela "Juventud del Sur" ubicada en la Gran Base de Misiones de Flor Amarillo en el estado Carabobo. Las bases teóricas utilizadas están fundamentadas en la visión del problema que asumen en la investigación los teóricos: Paulo Freire, Edgar Morín y Lev Vigotsky. La investigación fue de tipo proyectiva, bajo un diseño de campo. La técnica utilizada fue la encuesta de tipo cerrada y como instrumento un cuestionario compuesto por 9 ítems de contenido, los resultados obtenidos en relación a la encuesta aplicada, arrojaron como resultado que la totalidad de los estudiantes está de acuerdo con que es necesario el uso de talleres de formación alternativa para el aprendizaje, lo que permite la formación de estos jóvenes y contribuye a promover la revitalización de los programas de formación.

Palabras clave: programa; alternativo; estudiantes; base de misiones

\section{ABSTRACT}

The purpose of this article was to propose an alternative educational program to the students of the "Juventud for Sur" school located in the Great Yellow Flower Missions Base in Carabobo state. The theoretical bases used are based on the vision of the problem assumed by the theoreticians: Paulo Freire, Edgar Morin and Lev Vigotsky. The research was projective, under a field design. The technique used was the closedtype survey and as an instrument a questionnaire composed of 9 items of content, the results obtained in relation to the applied survey, showed that the totality of the students agrees that the use of Alternative training workshops for learning, which allows the training of these young people and helps to promote the revitalization of training programs.

Key words: program; alternative; students; mission base 


\section{INTRODUCCIÓN}

A lo largo de la historia, el hombre ha atravesado períodos de progreso y de cambios en su sociedad. Todas aquellas personas que están relacionadas con la educación que han sido a su vez instruidas de manera formal por una institución pueden reconocer la importancia que representa la escuela como promotor de valores y actitudes para una sociedad.

Actualmente escuchar sobre el mejoramiento de la "calidad" de manera educativa es común pero ¿Qué es la calidad? ¿Qué debe incluir?

Las respuestas variaran de acuerdo a cada institución. Algunas buscan que los estudiantes sean capaces de resolver problemas de una manera eficiente, donde los profesores impartan la información más reciente con técnicas modernas como el constructivismo. Otras pretenden educar en valores tradicionales de acuerdo a la religión que se profese en la institución. Por lo tanto se puede afirmar que el mejoramiento de calidad es subjetivo, ya que cada institución educativa tendrá una visión propia, razón que le da su particularidad a la oferta en la educación que se imparta en un colegio determinado.

Es esencial cumplir con los programas educativos alternativos ya que este ayuda al mejoramiento y mejor funcionamiento del sistema educativo. Por lo tanto, tanto educadores como las instituciones hacen hincapié en clases interactivas, lúdicas y atractivas donde el estudiante comprenda no solo los contenidos, sino que además sea capaz de llevarlo a su vida diaria.

El programa educativo alternativo que se implantó, se integra por asignaturas y talleres de carácter científico, humanístico, social y tecnológico, encaminados en la construcción a la formación integral de los estudiantes, es decir al desarrollo de sus áreas intelectual, afectiva, física, estética, artística, oral y de la comunicación.

Con este programa educativo alternativo y tomando en cuenta la escuela Juventud del Sur ubicada en la gran base de misiones de Flor Amarillo en el estado Carabobo y el Ministerio de Educación, para una formación integral, la propuesta que aquí se plantea pretendió fomentar las condiciones básicas, para ayudar a la toma de decisiones en un área no necesariamente humanista como lo son la ciencias naturales: fomentando un pensamiento divergente, es decir, personal y único, para que de esta forma los estudiantes puedan aceptar el compromiso de ser libres y responsables de tomar el camino correcto que crea conveniente para su vida. Es de notar que la gran mayoría de estos jóvenes estudiantes que asisten a la escuela juventud del sur, poseen un nivel de madurez muy avanzado para enfrentar la vida, esto dado por la imposición de responsabilidades del hogar y el enfrentamiento que tienen que hacerle a los duros momentos sociales y económicos que se les presenta día a día.

Estos aspectos fueron considerados complementarios a la hora de profundizar en como los estudiantes modifican sus conocimientos y procesos de pensamiento, consecuentemente estudiar sus implicaciones en el diseño de la instrucción puede considerarse una aportación integradora. Es decir empaparlos de conocimientos y obtener una evolución conceptual de los jóvenes estudiantes y la interacción que sobre todo el proceso va a tener las diferencias individuales entendidas desde una perspectiva cognitiva. Estos conocimientos implementados van a promover en los estudiantes una actitud positiva hacia el aprendizaje de cada uno de los contenidos y de la ciencia en general y hacia la resolución de problemas en particular.

Cabe destacar que los programas educativos alternativos son necesarios, porque 
ayudan a fortalecer las habilidades y destrezas de cada individuo permitiéndoles obtener de ellos los conocimientos necesarios para su formación personal. Es por ello que los contenidos que se implementen deben ser desarrollados para el conocimiento práctico, lo cual permitirá que los estudiantes tengan nivel educativo más completo.

Por ello, el objetivo general de este trabajo propone un Programa Educativo Alternativo para los niños y jóvenes de la Escuela "Juventud del Sur" ubicada en la Gran Base de Misiones de Flor Amarillo del Estado Carabobo. Y los objetivos específicos buscan describir un programa educativo alternativo integral para los niños y jóvenes de la Escuela "Juventud del Sur" ubicada en la Gran Base de Misiones de Flor Amarillo del Estado Carabobo; determinar un programa de formación alternativo para el trabajo que contemple el aprendizaje de un oficio útil para los niños y jóvenes de la Escuela "Juventud del Sur" ubicada en la Gran Base de Misiones de Flor Amarillo del Estado Carabobo y analizar un programa de clases que involucren historia y geografía de Venezuela pero impartidas a través del método geo-histórico para los niños y jóvenes de la Escuela "Juventud del Sur" ubicada en la Gran Base de Misiones de Flor Amarillo del Estado Carabobo.

\section{A manera de sustento teórico}

Paulo Freire (2002) refiriéndose a la Educación alternativa indica la base de su pensamiento es que no se puede negar a las personas el derecho a serlo plenamente. Es decir, a ser libres constructores de su propio futuro. Para ello, hay dos posibilidades en el frente educativo: o se está al servicio de la opresión del dominio, o se produce un proceso de liberación.

El autor se refiriere en que se debe tener iniciativa propia, buscando las herramientas pertinentes que se puedan ejecutar dentro de los programas educativos alternativos mediante un proceso educomunicativo, que emplee metodologías como la educación popular, la educación por el arte y la ludo pedagogía. Es por ello que los protagonistas deben ser los jóvenes adolescentes y se fomente la capacidad de creación de espacios libres, así como se ha venido llevando a cabo en la escuela "Juventud del Sur" ubicada en Flor Amarillo. Donde se está ejecutando el programa educativo alternativo. Donde ejercer sus derechos de una forma activa potenciando así la libre expresión y creación generando entornos propicios para adquirir aprendizajes significativos, basados entre las personas y su contexto.

Morín (1999) en el libro los siete saberes de la educación hace mención sobre los principios que garantice un conocimiento pertinente: existe un problema capital, aún desconocido, la necesidad de promover un conocimiento capaz de abordar problemas globales y fundamentales, para inscribir allí conocimientos parciales y locales. La supremacía de un conocimiento fragmentado según las disciplinas impiden a menudo operar el vínculo entre las partes y las totalidades y debe dar paso a un modo de conocimiento capaz de aprehender los objetos en sus contextos, sus complejidades, sus conjuntos.

Para Brembeck (2005) la educación debe ser ejecutada dentro del contexto global y multidimensional a través de los conocimientos y formas de enseñanzas, informaciones o elementos que no queden aislados, es decir, si se asume que en una propuesta de innovación educativa se desarrollará un proceso de reflexión, intervención y evaluación para mejorar la práctica educativa, se tiene claro cuáles son las dimensiones que deben tenerse en cuenta en la instauración y evaluación de esa innovación educativa, y en este sentido, es posible analizar la educación a través de los métodos educativos como el aprendizaje-servicio, que 
tengan como objetivo superar la pobreza, el racismo o cualquier forma de opresión y proponer dichas innovaciones de manera neutra, la respuesta es compleja pero se sabe que es necesaria para la formación de los estudiantes, es por eso que la escuela juventud del sur, tiene como finalidad canalizar y motivar a sus estudiantes de manera más práctica para su buen desenvolvimiento al momento en que deben ser evaluados.

Según Vergara (2017) Lev Vigotsky plantea que en el constructivismo social la mente $\mathrm{y}$ sus funciones superiores se originan en la interacción social. También menciona que los aprendizajes se producen en un contexto histórico y culturalmente determinado, que se aprende a través de su interacción con los demás, y se produce el desarrollo cuando internamente se controla el proceso, integrando nuevas competencias a la estructura cognitiva existente. Vigotsky considera el aprendizaje como uno de los mecanismos fundamental en la educación, en su opinión, la mejor enseñanza es la que se adelanta al desarrollo que se va ejecutando a través del modelo de aprendizaje que aporta, el contexto ocupa un lugar central, la interacción social se convierte en el motor del desarrollo educativo

Cabe destacar que en el enfoque constructivista, es utilizarlo como metodología de enseñanza para que los jóvenes de la escuela Juventud del Sur y todo aquel que puedan construir su propio conocimiento a través de su interacción y desenvolvimiento. El docente será el guía de su misma construcción. Por lo tanto, el nivel de su adaptación en tiempo y espacio dentro de una sociedad determinada puede adquirir conocimiento previo a través de su interacción con los estudiantes y así obtener grandes respuestas de los mismos y por supuesto obteniendo una estupenda retroalimentación.

Ahora bien, en cuanto a los beneficios de la participación socio-educativa de la familia, según Arocha (1997) la familia, conjuntamente con las comunidades educativas constituye una fuente inagotable de riqueza formativa, laboral y hasta económica; mediante ellas se obtiene apoyo en el proceso educativo, apoyo para la seguridad del plantel, apoyo laboral en caso de suplencias y hasta económico, formando profesionales que pueden colaborar. Son excelentes órganos para lograr y constituir un poderoso eslabón cogestor entre el liceo, la familia, comunidad y sin dejar pasar el Programa Educativo Alternativo para los Estudiantes de la Escuela Juventud del Sur.

La participación facilita el contacto personal entre los padres y los docentes, a través de las sugerencias e iniciativas de padres, docentes y estudiantes.

Como lo señala Albornoz (1984) para lograr buenos estudiantes, hay que tener buenos hijos y buenos padres, con familias armoniosas y funcionales. La interacción entre la familia y el liceo constituye el nudo central para la educación de los hijos, que son los mismos estudiantes. Por consiguiente, para este beneficio en particular, se nota la necesidad de formar a los padres y la escuela de padres, ya que este juega el rol principal para esta función como un eficaz proceso de integración. En el programa educativo alternativo para los estudiantes de la escuela juventud del sur, también se dictan talleres a los familiares de los estudiantes que hacen vida en dicha escuela, motivándolos a la integración educativa y pedagógica, con el reforzamiento mutuo del proceso de aprendizaje vivencias y valores. Por lo tanto, todo centro educativo que busque la calidad y la excelencia educativa ha de priorizar como eje central de su gerencia social la integración entre escuela familia y comunidad. Lograr la excelencia y la tan anhelada calidad educativa será prácticamente imposible sin la integración. Además, la participación permite, la identificación e involucración real del docente, del estudiante, de 
los padres, representantes y de todo el personal. Integración es identificación, es orgullo, es tradición, es cultura institucional.

\section{MÉTODO}

Se puede decir que el tipo y diseño de la investigación, basado en lo expuesto al respecto por Hernández, Fernández, y Baptista (2006) es de tipo proyectiva, bajo un diseño de cualidades y a la vez de campo. Que va dirigido a los jóvenes estudiantes de la escuela "Juventud del Sur" ubicada en la Gran Base de Misiones de Flor Amarillo. Con el fin de adaptar el lenguaje para trabajar la formación de la juventud; deben pensar en nuevas metodologías que permitan el desarrollo de la conciencia, el dinamismo, la creatividad y la organización de los mismos. La propuesta parte de la necesidad de generar un espacio que brinde las condiciones para la discusión, la reflexión crítica y profunda de lo social como concepto y como actividad.

En la investigación se utilizó la técnica, encuesta, a través de ellas se recabó información necesaria de los estudiantes, frente al proceso de enseñanza aprendizaje de los mismos.

En dicho programa educativo alternativo se utilizó la técnica que no es más que una encuesta de tipo cerrada y como instrumento un cuestionario compuesto por 9 ítems, lo cual es totalmente confidencial cuyos resultados se dieron a conocer únicamente en forma tabulada e impersonal, se buscó la reflexión efectiva de propuesta, proyectos sociales y educativos, rescatar, difundir y llevar a la práctica de los saberes, experiencia y tecnología de los estudiantes y toda aquella persona que hacen vida en dicha escuela, para integrarlos a los programas de formación general $\mathrm{y}$ fortalecer el acceso cultural, regional y nacional.

La población objeto de estudio está conformada de 26 jóvenes estudiantes que hacen vida en la escuela "Juventud del Sur" ubicada en la Gran Base de Misiones de Flor Amarillo en el Edo. Carabobo. Tal como lo plantea al respecto Hurtado (2002) la muestra estará representada por 26 estudiantes, debido a que es un programa que ya se está ejecutando en la escuela "Juventud del Sur", sin embargo aún no se existe una cantidad de educandos, por lo que se tomó la totalidad de estudiantes con muestra para la investigación.

Luego del análisis estadístico pertinente aplicado a los resultados obtenidos a través del instrumento utilizado en la investigación; se puede visualizar en formas de tablas distribuidas según frecuencias e índices porcentuales acompañados de gráficos lo cual facilitará la compresión de la información en relación a este planteamiento.

Tabla 1. Talleres de formación alternativa para el aprendizaje de un oficio útil

\begin{tabular}{ccccccc}
\hline & \multicolumn{4}{c}{ Sexo } & \multirow{2}{*}{ TOTAL } \\
\cline { 2 - 6 } & \multicolumn{1}{c}{ Masculino } & Femenino & & \\
\cline { 2 - 6 } & $\mathbf{f}$ & $\mathbf{\%}$ & $\mathbf{f}$ & $\mathbf{\%}$ & $\mathbf{f}$ & $\mathbf{\%}$ \\
\hline SI & 11 & $42 \%$ & 15 & $58 \%$ & 26 & $100 \%$ \\
NO & 0 & $0 \%$ & 0 & $0 \%$ & 0 & $0 \%$ \\
\hline TOTAL & $\mathbf{1 1}$ & $\mathbf{4 2 \%}$ & $\mathbf{1 5}$ & $\mathbf{5 8 \%}$ & $\mathbf{2 6}$ & $\mathbf{1 0 0 \%}$ \\
\hline
\end{tabular}

Fuente: Encuesta aplicada a los jóvenes estudiantes de la escuela "Juventud del Sur" 


\section{Interpretación}

La encuesta aplicada a los jóvenes estudiantes de la escuela "Juventud del Sur" ubicada en la gran base de misiones de Flor Amarillo del estado Carabobo arrojo como resul- tado que el $100 \%$ está en total acuerdo con que es necesario el uso de talleres de formación alternativa para el aprendizaje de un oficio útil.

Tabla 2. Propuesta de un programa educativo alternativo tiene mucha importancia en la actualidad.

\begin{tabular}{ccccccc}
\hline & \multicolumn{4}{c}{ Sexo } & \multirow{2}{*}{ TOTAL } \\
\cline { 2 - 5 } & \multicolumn{4}{c}{ Masculino } & \multicolumn{1}{c}{ Femenino } & \\
\cline { 2 - 6 } & $\mathbf{f}$ & $\mathbf{\%}$ & $\mathbf{f}$ & $\mathbf{\%}$ & $\mathbf{f}$ & $\mathbf{\%}$ \\
\hline SI & 8 & $30 \%$ & 14 & $54 \%$ & 22 & $84 \%$ \\
NO & 3 & $12 \%$ & 1 & $4 \%$ & 4 & $16 \%$ \\
\hline TOTAL & $\mathbf{1 1}$ & $\mathbf{4 2 \%}$ & $\mathbf{1 5}$ & $\mathbf{5 8 \%}$ & $\mathbf{2 6}$ & $\mathbf{1 0 0 \%}$ \\
\hline
\end{tabular}

Fuente: Encuesta aplicada a los jóvenes estudiantes de la escuela "Juventud del Sur"

\section{Interpretación}

Los resultados obtenidos indicaron que un $84 \%$ de los estudiantes encuestados dijeron que SI, de los cuales $30 \%$ son hombres y $54 \%$ mujeres, estando de acuerdo con que la propuesta de un programa educativo alternativo tiene mucha importancia en la actualidad, por otro lado un $16 \%$ dijo que NO, de los cuales $12 \%$ son hombres y $4 \%$ mujeres.

Tabla 3. Rendimiento académico desde que asiste a los talleres impartidos en la escuela juventud del sur

\begin{tabular}{ccccccc}
\hline & \multicolumn{4}{c}{ Sexo } & \multirow{2}{*}{ TOTAL } \\
\cline { 2 - 5 } & \multicolumn{2}{c}{ Masculino } & \multicolumn{2}{c}{ Femenino } & & \\
\cline { 2 - 6 } & $\mathbf{f}$ & $\mathbf{\%}$ & $\mathbf{f}$ & $\mathbf{\%}$ & f & $\mathbf{\%}$ \\
\hline SI & 11 & $42 \%$ & 15 & $58 \%$ & 26 & $100 \%$ \\
NO & 0 & $0 \%$ & 0 & $0 \%$ & 0 & $0 \%$ \\
\hline TOTAL & $\mathbf{1 1}$ & $\mathbf{4 2 \%}$ & $\mathbf{1 5}$ & $\mathbf{5 8 \%}$ & $\mathbf{2 6}$ & $\mathbf{1 0 0 \%}$ \\
\hline
\end{tabular}

Fuente: Encuesta aplicada a los jóvenes estudiantes de la escuela "Juventud del Sur"

\section{Interpretación}

El 100\% estudiantes de la escuela "Juventud del Sur" está en total acuerdo con que ha existido una mejoría en su rendimiento académico desde que asiste a los talleres impartidos en la escuela "Juventud del Sur". 
Tabla 4. Los talleres de formación alternativa impartidos en la escuela juventud del sur están siendo correctamente desarrollados.

\begin{tabular}{ccccccc}
\hline & \multicolumn{4}{c}{ Sexo } & \multirow{2}{*}{ TOTAL } \\
\cline { 2 - 7 } & Masculino & Femenino & & \\
\cline { 2 - 7 } & f & $\mathbf{\%}$ & f & \% & f & \% \\
\hline SI & 11 & $42 \%$ & 15 & $58 \%$ & 26 & $100 \%$ \\
NO & 0 & $0 \%$ & 0 & $0 \%$ & 0 & $0 \%$ \\
\hline TOTAL & $\mathbf{1 1}$ & $\mathbf{4 2 \%}$ & $\mathbf{1 5}$ & $\mathbf{5 8 \%}$ & $\mathbf{2 6}$ & $\mathbf{1 0 0 \%}$ \\
\hline
\end{tabular}

Fuente: Encuesta aplicada a los jóvenes estudiantes de la escuela "Juventud del Sur"

\section{Interpretación}

Los resultados muestran que la totalidad de los estudiantes de la escuela "Juventud del
Sur" creen que los talleres de formación alternativa impartidos están siendo correctamente desarrollados.

Tabla 5. Talleres para fomentar los hábitos de estudio desde el hogar.

\begin{tabular}{ccccccc}
\hline & \multicolumn{4}{c}{ Sexo } & \multirow{2}{*}{ TOTAL } \\
\cline { 2 - 7 } & Masculino & Femenino & & \\
\cline { 2 - 7 } & $\mathbf{f}$ & $\mathbf{\%}$ & $\mathbf{f}$ & $\mathbf{\%}$ & f & $\mathbf{\%}$ \\
\hline SI & 3 & $12 \%$ & 6 & $23 \%$ & 9 & $35 \%$ \\
NO & 8 & $30 \%$ & 9 & $35 \%$ & 17 & $65 \%$ \\
\hline TOTAL & $\mathbf{1 1}$ & $\mathbf{4 2 \%}$ & $\mathbf{1 5}$ & $\mathbf{5 8 \%}$ & $\mathbf{2 6}$ & $\mathbf{1 0 0 \%}$ \\
\hline
\end{tabular}

Fuente: Encuesta aplicada a los jóvenes estudiantes de la escuela "Juventud del Sur"

\section{Interpretación}

Los resultados conseguidos revelan que un $35 \%$ de los estudiantes encuestados dijeron que SI, de los cuales $12 \%$ son hombres y $23 \%$ mujeres, por otro lado un $65 \%$ dijo que
NO, de los cuales $30 \%$ son hombres y $35 \%$ mujeres, estando en contra con la idea de que los representantes formen también parte de los talleres para fomentar los hábitos de estudio desde el hogar.

Tabla 6. Implementar nuevas estrategias en el programa educativo alternativo de la escuela Juventud del Sur

\begin{tabular}{ccccccc}
\hline & \multicolumn{4}{c}{ Sexo } & \multirow{2}{*}{ TOTAL } \\
\cline { 2 - 7 } & Masculino & \multicolumn{1}{c}{ Femenino } & \multicolumn{1}{c}{} \\
\hline SI & $\mathbf{\%}$ & f & $\mathbf{\%}$ & f & $\%$ \\
NO & 9 & $34 \%$ & 11 & $42 \%$ & 20 & $76 \%$ \\
\hline TOTAL & $\mathbf{1 1}$ & $8 \%$ & 4 & $16 \%$ & 6 & $24 \%$ \\
\hline
\end{tabular}

Fuente: Encuesta aplicada a los jóvenes estudiantes de la escuela "Juventud del Sur" 


\section{Interpretación}

Los resultados recabados muestran que un $76 \%$ de los estudiantes encuestados dijeron que SI, de los cuales $34 \%$ son hombres y $42 \%$ mujeres, estando de acuerdo con la im- plementación de nuevas estrategias de aprendizaje en la escuela "Juventud del Sur". Por otro lado un $24 \%$ dijo que NO, de los cuales $8 \%$ son hombres y $16 \%$ mujeres.

Tabla 7. Talleres realizados te ha parecido más útil para tu vida

\begin{tabular}{ccccccc}
\hline \multirow{2}{*}{ Talleres } & \multicolumn{4}{c}{ Sexo } & \multicolumn{2}{c}{ TOTAL } \\
& \multicolumn{3}{c}{ Masculino } & Femenino & \\
\cline { 2 - 7 } & $\mathbf{f}$ & $\mathbf{\%}$ & $\mathbf{f}$ & $\mathbf{\%}$ & $\mathbf{f}$ & $\mathbf{\%}$ \\
\hline Nociones básicas de programación & 1 & $4 \%$ & 0 & $0 \%$ & 1 & $4 \%$ \\
Las Criptomonedas & 2 & $8 \%$ & 1 & $4 \%$ & 3 & $12 \%$ \\
Mediática en las redes sociales & 2 & $8 \%$ & 2 & $8 \%$ & 4 & $16 \%$ \\
Acoso por redes sociales & 0 & $0 \%$ & 4 & $14 \%$ & 4 & $14 \%$ \\
Problemas de salud publica & 2 & $8 \%$ & 3 & $12 \%$ & 5 & $20 \%$ \\
Crisis Alimentaria & 4 & $14 \%$ & 5 & $19 \%$ & 9 & $33 \%$ \\
\hline TOTAL & $\mathbf{1 1}$ & $\mathbf{4 2 \%}$ & $\mathbf{1 5}$ & $\mathbf{5 8 \%}$ & $\mathbf{2 6}$ & $\mathbf{1 0 0 \%}$ \\
\hline
\end{tabular}

Fuente: Encuesta aplicada a los jóvenes estudiantes de la escuela "Juventud del Sur"

\section{Interpretación}

Los resultados obtenidos revelan que la mayoría de los estudiantes encuestados, específicamente un $33 \%$, le ha parecido más útil el taller de "Crisis alimentaria, escasez de recursos, crisis económica" de los cuales un
$14 \%$ son hombres y $19 \%$ mujeres. Por el contrario, solo al $4 \%$ le ha parecido más útil el taller de "Nociones básicas de programación, big data e inteligencia artificial" siendo en su totalidad hombres.

Tabla 8. Talleres impartidos a través del programa educativo alternativo dados en la escuela Juventud del Sur te han parecido los más necesarios

\begin{tabular}{ccccccc}
\hline \multirow{2}{*}{ Talleres } & \multicolumn{4}{c}{ Sexo } & \multicolumn{2}{c}{ TOTAL } \\
\cline { 2 - 7 } & Masculino & Femenino & \\
\cline { 2 - 7 } & $\mathbf{f}$ & $\mathbf{\%}$ & $\mathbf{f}$ & $\mathbf{\%}$ & $\mathbf{f}$ & $\mathbf{\%}$ \\
\hline Nociones básicas de programación & 2 & $8 \%$ & 0 & $0 \%$ & 2 & $8 \%$ \\
Las Criptomonedas & 1 & $4 \%$ & 2 & $8 \%$ & 3 & $12 \%$ \\
Mediática en las redes sociales & 2 & $8 \%$ & 4 & $14 \%$ & 6 & $22 \%$ \\
Acoso por redes sociales & 0 & $0 \%$ & 4 & $14 \%$ & 4 & $14 \%$ \\
Problemas de salud publica & 2 & $8 \%$ & 2 & $8 \%$ & 4 & $16 \%$ \\
Crisis Alimentaria & 4 & $14 \%$ & 3 & $12 \%$ & 7 & $26 \%$ \\
\hline TOTAL & $\mathbf{1 1}$ & $\mathbf{4 2 \%}$ & $\mathbf{1 5}$ & $\mathbf{5 8 \%}$ & $\mathbf{2 6}$ & $\mathbf{1 0 0 \%}$ \\
\hline
\end{tabular}

Fuente: Encuesta aplicada a los jóvenes estudiantes de la escuela "Juventud del Sur" 


\section{Interpretación}

El 26\% de los estudiantes encuestados, de los cuales $14 \%$ son hombres y $12 \%$ son mujeres considera que el taller de "Crisis alimentaria, escasez de recursos, crisis econó- mica" es el más necesario, mientras que solo al 8\% le ha parecido más necesario el taller de "Nociones básicas de programación, big data e inteligencia artificial", siendo hombres en su totalidad.

Tabla 9. Talleres impartidos a través del programa educativo alternativo dados en la escuela Juventud del Sur no has realizado y te gustaría hacer

\begin{tabular}{|c|c|c|c|c|c|c|}
\hline \multirow{3}{*}{ Talleres } & \multicolumn{4}{|c|}{ Sexo } & \multirow{2}{*}{\multicolumn{2}{|c|}{ TOTAL }} \\
\hline & \multicolumn{2}{|c|}{ Masculino } & \multicolumn{2}{|c|}{ Femenino } & & \\
\hline & f & $\%$ & f & $\%$ & $\mathbf{f}$ & $\%$ \\
\hline Nociones básicas de programación & 2 & $8 \%$ & 2 & $8 \%$ & 4 & $16 \%$ \\
\hline Las Criptomonedas & 4 & $14 \%$ & 2 & $8 \%$ & 6 & $22 \%$ \\
\hline Mediática en las redes sociales & 0 & $0 \%$ & 3 & $12 \%$ & 3 & $12 \%$ \\
\hline Acoso por redes sociales & 0 & $0 \%$ & 4 & $14 \%$ & 4 & $14 \%$ \\
\hline Problemas de salud publica & 3 & $12 \%$ & 1 & $4 \%$ & 4 & $16 \%$ \\
\hline Crisis Alimentaria & 2 & $8 \%$ & 3 & $12 \%$ & 5 & $20 \%$ \\
\hline TOTAL & 11 & $42 \%$ & 15 & $58 \%$ & 26 & $100 \%$ \\
\hline
\end{tabular}

Fuente: Encuesta aplicada a los jóvenes estudiantes de la escuela "Juventud del Sur"

\section{Interpretación}

El 22\% de los estudiantes encuestados, de los cuales $14 \%$ son hombres y $8 \%$ son mujeres considera que el taller de "criptomonedas; bitcoin su influencia y el petro" es uno de los que no han realizado pero les interesaría hacer, mientras que solo $12 \%$ no ha realizado pero le interesaría hacer el taller de "Mediática en las Redes Sociales y auge e influencias de poder", siendo mujeres en su totalidad.

\section{CONCLUSIONES}

Una vez realizado el análisis de los resultados, con respecto a describir un programa educativo alternativo integral para los niños y jóvenes de la escuela "Juventud del Sur" ubicada en la Gran Base de Misiones de Flor Amarillo del Estado Carabobo. Los resultados obtenidos indican que un $84 \%$ de los estudiantes encuestados si están de acuerdo con la propuesta de un programa educativo alternativo y opinan que es de mucha importancia en la actualidad, por otro lado un $16 \%$ no cree que sea de tanta importancia implementar un programa educativo alternativo, sin embargo el $100 \%$ de la población estudiantil ha mejorado su rendimiento académico a partir de comenzar a asistir a los talleres de la escuela "Juventud del Sur".

En cuanto determinar un programa de formación alternativo para el trabajo que contemple el aprendizaje de un oficio útil para los niños y jóvenes de la escuela "Juventud del Sur", los resultados de la encuesta realizada lograron comprobar que el $100 \%$ está en total acuerdo con que los talleres de formación alternativa impartidos en la escuela "Juventud del Sur" son una ayuda fundamental para el aprendizaje de un oficio útil.

Con relación a analizar un programa de clases que involucren historia y geografía de Venezuela pero impartidas a través del método geo-histórico para los niños y jóvenes de la escuela "Juventud del Sur", fueron planteados varios talleres que cumplan con el método geo-histórico y que sean de gran beneficio 
para los estudiantes, los cuales serán implementados a medida que vaya avanzando el programa.

En último orden, teniendo en cuenta el resultado de los análisis de las encuestas realizadas, se da respuesta a este proyecto, con respecto a identificar el grado de instrucción que poseen los estudiantes y cuál ha sido su mejoría académica desde que asisten a la escuela "Juventud del Sur", ya que cumplen un papel importante tanto en el ámbito educativo como en el laboral, dando como resultado una muestra satisfactoria dentro del mismo, proporcionándole a los jóvenes estudiantes la capacidad de ejercer un oficio útil y poder contribuir en el patrimonio familiar. Un grupo que ha sido y seguirán siendo atendidos por una gran diversidad de profesionales en sus respectivos campos, en grados de instrucción desde 6to grado hasta profesionales. En la escuela "Juventud del Sur" de Flor Amarillo se continuará formando a los estudiantes que deseen y se sientan comprometidos con ellos mismos para su formación, con la finalidad de reforzarlos y motivarlos en sus actividades diarias.

La propuesta que se presenta en este artículo parte de la necesidad de generar un espacio que brinde las condiciones adecuadas para la discusión, la reflexión crítica y profunda de lo social como concepto y como acti- vidad.

\section{REFERENCIAS}

Albornoz, O. (1.984). La Familia y la educación del venezolano. Caracas. Ediciones de la Biblioteca de la Universidad Central de Venezuela

Arocha, L. (1.997) Efectos de la Integración familiar en el rendimiento estudiantil de los jóvenes. Tesis de grado. Universidad de Carabobo. Valencia

Brembeck, C. (2005). Ambiente y rendimiento escolar: El alumno - en desventaja. Buenos Aires: Editorial Paídós

Freire, P. (1989). La educación como práctica de la libertad. Madrid: Siglo XXI

Freire, P. (1992): Pedagogía del oprimido. Madrid: Siglo XXI

Freire, P. (2002). Pedagogía de la esperanza. México: Siglo XX

Hernández, R.; Fernández, C. y Baptista, P. (2006) Metodología de la investigación. México, editorial Mc Graw-Hill

Hurtado, J. (2002) Metodología de la investigación Holística. Universidad de Antioquia

Morín, E. (1999) Los siete saberes necesarios para la educación del futuro. Recuperado de

http://www.ideassonline.org/public/pdf /LosSieteSaberesNecesariosParaLaEdude lFuturo.pdf

Vergara, C (2017) Vygotsky y la teoría sociocultural del desarrollo cognitivo, Recuperado de https://www.actualidadenpsicologia.com/vygotsky-teoria-sociocultur 